Proceedings

\title{
Ferromagnetic Josephson Junctions for High Performance Computation ${ }^{\dagger}$
}

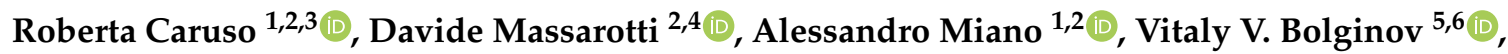 \\ Aymen Ben Hamida ${ }^{6,7}$ (D) Liubov N. Karelina ${ }^{5}$, Igor V. Vernik ${ }^{3,8}$, Valery V. Ryazanov ${ }^{5,9}$, \\ Oleg A. Mukhanov ${ }^{3,8}$, Giovanni Piero Pepe ${ }^{1,2}$ and Francesco Tafuri ${ }^{1,2, *}$ \\ 1 Dipartimento di Fisica "E. Pancini”, Università di Napoli Federico II, Monte Sant'Angelo, via Cinthia 26, \\ I-80126 Naples, Italy; caruso@fisica.unina.it (R.C.); amiano@fisica.unina.it (A.M.); gpepe@na.infn.it (G.P.P.) \\ 2 CNR-SPIN, c/o Monte Sant'Angelo, via Cinthia 26, I-80126 Naples, Italy; davide.massarotti@unina.it \\ 3 SeeQC-eu, via dei Due Macelli 66, I-00187 Rome, Italy; igorv@seeqc.eu (I.V.V.); olegm@seeqc.eu (O.A.M.) \\ 4 Dipartimento di Ingegneria Elettrica e delle Tecnologie dell'Informazione, Università di Napoli Federico II, \\ via Claudio 25, I-80125 Naples, Italy \\ 5 Institute of Solid State Physics (ISSP RAS), Chernogolovka, Moscow Region 142432, Russia; \\ bolg@mail.ru (V.V.B.); karelina@issp.ac.ru (L.N.K.); ryazanov@issp.ac.ru (V.V.R.) \\ 6 National University of Science and Technology MISIS, 4 Leninsky prosp., Moscow 119049, Russia; \\ benhamida.aymen@gmail.com \\ 7 Leiden Institute of Physics, Leiden University, Niels Bohrweg 2, 2333 CA Leiden, The Netherlands \\ 8 HYPRES, Inc., 175 Clearbrook Road, Elmsford, NY 10523, USA \\ 9 Faculty of Physics, National Research University Higher School of Economics, Moscow 105066, Russia; \\ * Correspondence: francesco.tafuri@unina.it \\ + Presented at the 11th Italian Quantum Information Science conference (IQIS2018), Catania, Italy, \\ 17-20 September 2018.
}

Published: 25 June 2019

\begin{abstract}
Josephson junctions drive the operation of superconducting qubits and they are the key for the coupling and the interfacing of superconducting qubit components with other quantum platforms. They are the only means to introduce non linearity in a superconducting circuit and offer direct solutions to tune the properties of a superconducting qubit, thus enlarging the possible qubit layouts. Junctions performances and tunability can take advantage of using a large variety of barriers and their special functionalities. We mention pertinent results on the advances in understanding the properties of ferromagnetic junctions, which make possible the use of these devices either as memory elements and as core circuit elements.
\end{abstract}

Keywords: Josephson junctions; ferromagnetic barriers; cryogenic memories; superconducting qubits

\section{Introduction}

The control of a Josephson junction (JJ) is meant to be more and more important for the development of novel concepts and layouts of superconducting elements for a wide range of applications, ranging from digital circuits to different types of superconducting qubits. If on one hand $\mathrm{Al}$ junctions technology needs to do further steps to guarantee a huge number of junctions with required yield and reproducibility for further advances in the realization of a quantum computer, on the other hand it is reasonable to expect that hybrid solutions may offer some alternatives in the realization of complex quantum systems. For hybrid we mean either junctions employing barriers other than common oxides such as $\mathrm{AlO}_{x}$, or physical systems which can incorporate various quantum systems in the same architecture. This hybrid perspective is the motivation behind the integration of a transmon in a cavity and a spin ensemble [1], or the realization of a gatemon [2,3]. An hybrid system for novel functionalities wants to take advantage of the various quantum components, while a hybrid 
junction wants to use more efficient knobs and controls, and this is not always possible in traditional $\mathrm{Al}$ and $\mathrm{Nb}$ technologies. This is operatively possible because some of these circuits, as for instance the transmon, do not necessarily require to use junctions with very high quality factors.

Keeping in mind that a 'different' junction can offer novel functionalities or is more easily prone to be controlled by alternative and possibly less noisy knobs, or to be coupled to other platforms, we will describe some interesting properties of ferromagnetic SFS (Superconductor-Ferromagnet-Superconductor) junctions which pave the way to their use in real quantum circuits. In addition to the well established notion of using SFS junctions as energy-efficient Random Access Memories (RAM) to be employed for hybrid quantum/classic high performance computation, we report on the demonstration of how an $\mathrm{RF}$ train can change the state of the memory suggesting an RF drive for SFS circuits in some specific conditions. The notions developed for these types of junctions could be in the long run used as a new tool to control the state of a transmon, more easily connected to a qubit control driven by Single Flux Quantum (SFQ) logic [4].

\section{Memory Properties of Ferromagnetic Junctions}

In the last few years, there have been several approaches to the use of the memory properties of ferromagnetic JJs. Most recent achievements employ spin valves [5] or pseudo-spin valves [6] as junction barriers to control the phase or the critical current magnitude switch between two well-defined states. Another interesting approach uses $\varphi$-junctions as memory elements, defining the two logic states as the escape from the two different wells of the degenerate washboard potential, which is characteristic for such devices [7].

The possibility to use ferromagnetic JJs as RAMs was demonstrated by Bol'ginov et al. [8]. In these $\mathrm{Nb}-\mathrm{PdFe}-\mathrm{Nb}$ trilayers, the two classical logic states correspond to two different critical current levels due to the hysteretic behavior of ferromagnetic materials. Such materials have a multivalued dependence of the magnetization $\mathbf{M}$ upon the external magnetic field $\mathbf{H}$, which is reflected on the critical current dependence of the junction on the external magnetic field (Figure 1a). The use of positive and negative external magnetic field pulses allows to switch between the two critical current states (Figure $1 b, c)$. The readout is performed by applying a reading current $I_{\text {read }}$ such that the condition $I_{1}<I_{\text {read }}<I_{2}$ is satisfied, where $I_{1}$ and $I_{2}$ are the two critical current levels, and by measuring the voltage across the junction. If the device is in the state with $I_{C}=I_{1}$, then the output is a finite voltage, while if it is in the state with $I_{C}=I_{2}$ no voltage is detected across the junction.

The junctions used to demonstrate the magnetic switch have a metallic barrier, so their dissipation is high when compared with standard SIS tunnel junctions. While for SIS junctions typical values of $I_{C} R_{N}$ are as high as $1 \mathrm{mV}$ and the junctions are underdamped, typical values of $I_{C} R_{N}$ in metallic junctions are in the range of tens of $\mathrm{nV}$ and the junctions fall in the overdamped regime with quality factor $Q<<1$. In terms of compatibility with existing superconducting electronics, especially with SFQ circuits, this represents a major disadvantage, as in this case the voltage levels are typically of the order of millivolts. Recently, dissipation in spin valve JJs with Ho/Co barriers has been fully classified within the existing models to describe a JJ [9].

A significant step towards a full compatibility between superconducting magnetic RAM (MRAM) and SFQ circuits, and in general towards the use of low dissipation memory devices with high voltage levels, has been reported by Larkin et al. in 2012 [10], by using a modification of the SFS junction characterized in [8], with a more complex barrier. This includes an insulating $\mathrm{Al} / \mathrm{AlO}_{x}$ layer, a thin superconducting $\mathrm{Nb}$ interlayer, and a ferromagnetic PdFe layer. Such stacked barrier allows to obtain voltage levels comparable with the ones typical of SFQ circuits, keeping the switching properties intact. Junctions using ferromagnetic insulating barriers as GdN [11], completely falling in the underdamped regime and even showing macroscopic quantum tunneling effects [12] and indication of spin-triplet supercurrents [13], have also shown properties in principle compatible with memory applications. 

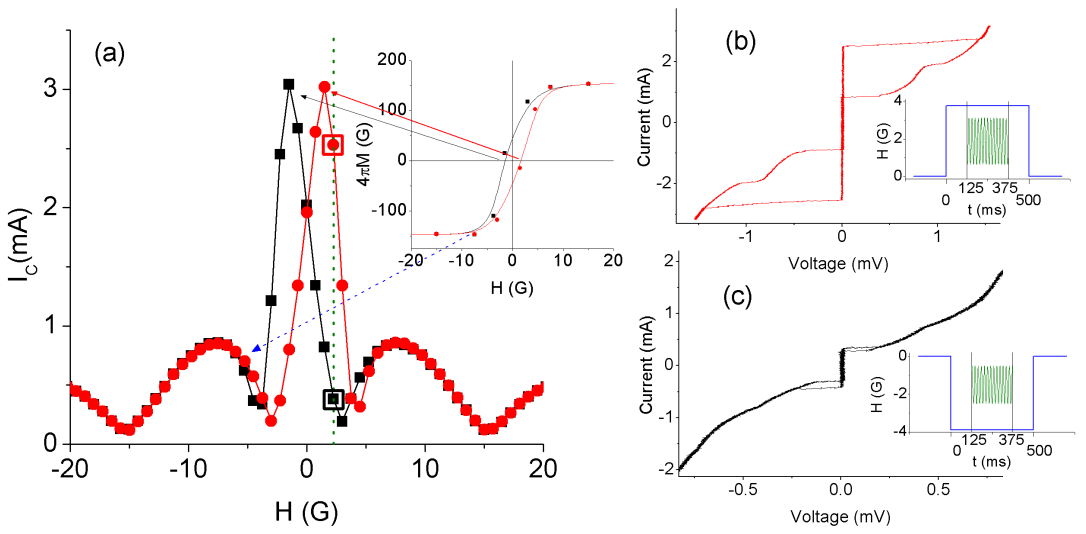

Figure 1. (a) $I_{C}(H)$ at $3.5 \mathrm{~K}$. Black squares correspond to an external magnetic field ramped from positive to negative values, red circles are obtained ramping the field from negative to positive values. Lines are plotted as a guide for the eye. Green dotted line represents the chosen working point. Inset: magnetization curve for the same sample, obtained using the technique described in [8]. Red and black arrows indicate the positive and negative coercive field $H_{C}$ on the two curves. Blue arrow indicates the saturation field $H_{S}$ on both curves. $(\mathbf{b}, \mathbf{c}) I(V)$ curves corresponding to the points highlighted by green dotted line in panel (a), which are the two critical current levels used as logical states. The insets show the combination of magnetic field pulses and RF trains used to induce the switch between the two states.

Recent experiments on SIsFS junctions have demonstrated that the switching mechanism can be enhanced by using external RF fields [14,15]. This result is of relevance because it indicates an additional tool to manipulate the memory state more efficiently. The effect of RF fields on the magnetization loops of ferromagnetic materials has been extensively studied in several other systems [14,16], however, this represents the first attempt on magnetic JJs. We have found that the current level separation enhancement depends on the magnetic field pulse amplitude, on the total energy of the RF train and on the working temperature. The dependence on the magnetic field pulse amplitude is due to the magnetization curve of the ferromagnet: if the pulses are close to the saturation field, then any change induced by the RF field is covered by the saturation, while if the pulse amplitude is too close to the working point, the enhancement is comparable to the current level fluctuations and so it is not significant. The largest effect is observed for intermediate pulse amplitudes, where the slope of the $M(H)$ curve allows to appreciate the change in the magnetization induced by the microwaves (see Figure 1a, inset). By investigating the enhancement for different power levels and duration of the RF train, we see that the relevant parameter is the total energy associated with the microwaves, rather than the power level or the duration alone. This indicates that the external RF field induces fluctuations in the ferromagnet that are analogous to thermal fluctuations. Finally, we analyzed the thermal behavior of the current level separation enhancement, and we found it compatible with existing literature on PdFe magnetic structure [17]: we observe a larger effect in specific temperature ranges, where one of the two mechanisms that cause ferromagnetism in PdFe can be switched off by the fluctuations induced by the microwaves. This latter result confirms the assumption that the fluctuations induced in PdFe by RF fields are analogous to thermal fluctuations.

The use of RF fields to control the critical current of magnetic Josephson junctions adds to the well-established capabilities to use ferromagnetic junctions for phase qubits [18-20].

\section{Conclusions and Perspectives}

The tunability of the critical current in different types of ferromagnetic junctions leading to their memory capabilities make them possibly useful in the long range not only for the integration in SFQ circuits, but also for the development of specific types of qubits. Apart from the phase qubit [18-20], a transmon could benefit of the notions developed for ferromagnetic JJs. The ratio $E_{J} / E_{C}$ would 
be no longer tuned by a dc-SQUID, but using a single magnetic JJ (MJJ), via magnetic pulses or $\mathrm{RF}$ trains. The memory properties of ferromagnetic Josephson junctions enable them to retain their state (and so their Josephson energy) when the external field is removed, once they are initialized before all the qubit operations, down to typical qubit operational temperatures. By changing the control input, it is possible to obtain different critical current levels and so different $E_{J} / E_{C}$ ratios. In Figure 2a, we show the different critical current levels obtained using RF trains with different nominal energies. The problem of coherence time and dissipation is obvioulsy complex for all types of qubits and not under control [21-23]. The architecture qualitatively sketched in Figure $2 b$ in parallel with the capacitive shunting connection $C_{B}$ typical of a transmon qubit, could reduce noise and decoherence associated with the presence of a static magnetic field and corresponding control lines at low temperatures.

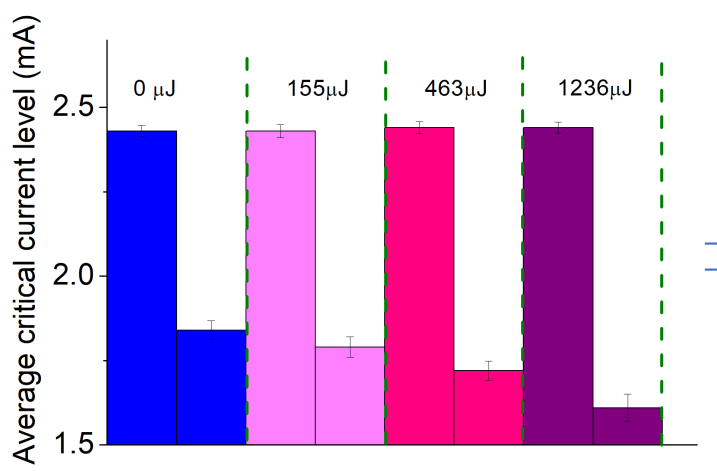

(a)

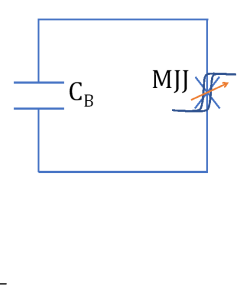

(b)

Figure 2. (a) Critical current levels obtained using RF trains with different nominal energies. (b) Sketch of the MJJ in parallel with the shunting capacitance $C_{B}$ typical of the transmon design.

Acknowledgments: The authors thank NANOCOHYBRI project (Cost Action CA 16218).

Conflicts of Interest: The authors declare no conflict of interest.

\section{References}

1. Xiang, Z.L.; Ashhab, S.; You, J.Q.; Nori, F. Hybrid quantum circuits: Superconducting circuits interacting with other quantum systems. Rev. Mod. Phys. 2013, 85, 623. doi:10.1103/RevModPhys.85.623.

2. Larsen, T.W.; Petersson, K.D.; Kuemmeth, F.; Jespersen, T.S.; Krogstrup, P.; Nygård, J.; Marcus, C.M. Semiconductor-Nanowire-Based Superconducting Qubit. Phys. Rev. Lett. 2015, 115, 127001. doi:10.1103/PhysRevLett.115.127001.

3. De Lange, G.; van Heck, B.; Bruno, A.; van Woerkom, D.J.; Geresdi, A.; Plissard, S.R.; Bakkers, E.P.A.M.; Akhmerov, A.R.; DiCarlo, L. Realization of Microwave Quantum Circuits Using Hybrid Superconducting-Semiconducting Nanowire Josephson Elements. Phys. Rev. Lett. 2015, 115, 127002. doi:10.1103/PhysRevLett.115.127002.

4. Leonard, E., Jr.; Beck, M.A.; Nelson, J.J.; Christensen, B.G.; Thorbeck, T.; Howington, C.; Opremcak, A.; Pechenezhskiy, I.V.; Dodge, K.; Dupuis, N.P.; et al. Digital coherent control of a superconducting qubit. arXiv 2019, arXiv:1806.07930.

5. $\quad$ Gingrich, E.C.; Niedzielski, B.M.; Glick, J.A.; Wang, Y.; Miller, D.L.; Loloee, R.; Pratt, W.P., Jr.; Birge, N.O. Controllable $0-\pi$ Josephson junctions containing a ferromagnetic spin valve. Nat. Phys. 2016, 12, $564-567$. doi:10.1038/nphys3681.

6. Baek, B.; Rippard, W.H.; Benz, S.P.; Russek, S.E.; Dresselhaus, P.D. Hybrid superconducting-magnetic memory device using competing order parameters. Nat. Commun. 2014, 5, 4888. doi:10.1038/ncomms4888.

7. Goldobin, E.; Sickinger, H.; Weides, M.; Ruppelt, N.; Kohlstedt, H.; Kleiner, R.; Koelle, D. Memory cell based on a $\varphi$ Josephson junction. Appl. Phys. Lett. 2013, 102, 242602. doi:10.1063/1.4811752. 
8. Bol'ginov, V.V.; Stolyarov, V.S.; Sobanin, D.S.; Karpovich, A.L.; Ryazanov, V.V. Magnetic switches based on $\mathrm{Nb}-\mathrm{PdFe}-\mathrm{Nb}$ Josephson junctions with a magnetically soft ferromagnetic interlayer. JETP Lett. 2012, 95, 366-371. doi:10.1134/S0021364012070028.

9. Massarotti, D.; Banerjee, N.; Caruso, R.; Rotoli, G.; Blamire, M.G.; Tafuri, F. Electrodynamics of Josephson junctions containing strong ferromagnets. Phys. Rev. B 2018, 98, 144516. doi:10.1103/PhysRevB.98.144516.

10. Larkin, T.I.; Bol'ginov, V.V.; Stolyarov, V.S.; Ryazanov, V.V.; Vernik, I.V.; Tolpygo, S.K.; Mukhanov, O.A. Ferromagnetic Josephson switching device with high characteristic voltage. Appl. Phys. Lett. 2012, 100, 222601. doi:10.1063/1.4723576.

11. Senapati, K.; Blamire, M.G.; Barber, Z.H. Spin-filter Josephson junctions. Nat. Mater. 2011, 10, 849-852. doi:10.1038/nmat3116.

12. Massarotti, D.; Pal, A.; Rotoli, G.; Longobardi, L.; Blamire, M.G.; Tafuri, F. Macroscopic quantum tunnelling in spin filter ferromagnetic Josephson junctions. Nat. Commun. 2015, 6, 7376. doi:10.1038/ncomms8376.

13. Caruso, R.; Massarotti, D.; Campagnano, G.; Pal, A.; Ahmad, H.G.; Lucignano, P.; Eschrig, M.; Blamire, M.G.; Tafuri, F. Tuning of magnetic activity in spin-filter Josephson junctions towards spin-triplet transport Phys. Rev. Lett. 2019, 122, 047002. doi:10.1103/PhysRevLett.122.047002.

14. Caruso, R.; Massarotti, D.; Bolginov, V.V.; Hamida, A.B.; Karelina, L.N.; Miano, A.; Vernik, I.V.; Tafuri, F.; Ryazanov, V.V.; Mukhanov, O.A.; et al. RF assisted switching in magnetic Josephson junctions. J. Appl. Phys. 2018, 123, 133901. doi:10.1063/1.5018854.

15. Caruso, R.; Massarotti, D.; Miano, A.; Bolginov, V.V.; Hamida, A.B.; Karelina, L.N.; Campagnano, G.; Vernik, I.V.; Tafuri, F.; Ryazanov, V.V.; et al. Properties of Ferromagnetic Josephson Junctions for Memory Applications. IEEE Trans. Appl. Supercond. 2018, 28, 1800606. doi:10.1109/TASC.2018.2836979.

16. Thirion, C.; Wernsdorfer, W.; Mailly, D. Switching of magnetization by nonlinear resonance studied in single nanoparticles. Nat. Mater. 2003, 2, 524-527. doi:10.1038/nmat946.

17. Bol'ginov, V.V.; Tikhomirov, O.A.; Uspenskaya, L.S. Two-component magnetization in Pd99Fe01 thin films. JETP Lett. 2017, 105, 169-173. doi:10.1134/S0021364017030055.

18. Shcherbakova, A.V.; Fedorov, K.G.; Shulga, K.V.; Ryazanov, V.V.; Bolginov, V.V.; Oboznov, V.A.; Egorov, S.V.; Shkolnikov, V.O.; Wolf, M.J.; Beckmann, D. Fabrication and measurements of hybrid Nb/Al Josephson junctions and flux qubits with $\pi$-shifters Supercond. Sci. Technol. 2015, 28, 025009. doi:10.1088/0953-2048.

19. Feofanov, A.K.; Oboznov, V.A.; Bol'ginov, V.V.; Lisenfeld, J.; Poletto, S.; Ryazanov, V.V.; Rossolenko, A.N.; Khabipov, M.; Balashov, D.; Zorin, A.B.; et al. Implementation of superconductor/ferromagnet/ superconductor $\phi$-shifters in superconducting digital and quantum circuits. Nat. Phys. 2010, 6, 593-597. doi:10.1038/nphys1700.

20. Kato, T.; Golubov, A.A.; Nakamura, Y. Decoherence in a superconducting flux qubit with a $\pi$-junction. Phys. Rev. B 2007, 76, 172502. doi:10.1103/PhysRevB.76.172502.

21. Schreier, J.A.; Houck, A.A.; Koch, J.; Schuster, D.I.; Johnson, B.R.; Chow, J.M.; Gambetta, J.M.; Majer, J.; Frunzio, L.; Devoret, M.H.; et al. Suppressing charge noise decoherence in superconducting charge qubits. Phys. Rev. B 2008, 77, 180502. doi:10.1103/PhysRevB.77.180502.

22. Clarke, J.; Wilhelm, F.K. Superconducting quantum bits. Nature 2008, 453, 1031-1042. doi:10.1038/nature07128.

23. Devoret, M.H.; Schoelkopf, R.J. Superconducting Circuits for Quantum Information: An Outlook. Science 2013, 339, 1169-1174.

(C) 2019 by the authors. Licensee MDPI, Basel, Switzerland. This article is an open access article distributed under the terms and conditions of the Creative Commons Attribution (CC BY) license (http:/ / creativecommons.org/licenses/by/4.0/). 